\title{
Management and archiving system for metal detection robot using wireless-based technology and online database registry
}

\author{
Hakim Adil Kadhim ${ }^{1}$, Nabeel Salih Ali ${ }^{2}$, Dheyaa M. Abdulsahib ${ }^{3}$ \\ ${ }^{1,3}$ Department of Electronic and Communications Engineering, University of Kufa, Iraq \\ ${ }^{2}$ Information Technology Research and Development Centre, University of Kufa, Iraq
}

\begin{tabular}{l} 
Article Info \\
\hline Article history: \\
Received Jul 9, 2018 \\
Revised Oct 4, 2018 \\
Accepted Nov 3, 2018 \\
\hline
\end{tabular}

Keywords:

Achieving system

Database

Information management

system

Metal detector

Multi-sensory robot system

Robot

Small-reaction manipulator

Vehicle-mounted sensors

\begin{abstract}
The tremendous recent involvement of technology in our life generates a lot of advantages and disadvantages. Nevertheless, and to profoundly augment its positive influence, at the expense of the negatives, most technology be deployed to serve humanity and society. Researchers attractive in developing robust and cooperative robotics that capable of solving difficult tasks without any human control. Metal detector robot is one of the robotics principles due to its effectiveness as compared with manually operated and very slow traditional methods. In this article, three main points that are concentrated 1) Design a robot which is vehicle-mounted sensors that capable of carries the sensors of the metal and obstacle; 2) Control and management system wirelessly by a computer-based to command the robot functions by several sets of user's rules and manage the robot instructions; and 3) Conduct an integrated system that achieving navigated data via metal detector based on online structured query language database registry. Also, discussed a comparison of the previous detector systems and highlights on several merits. The proposed system capable of fully control the robot also, set the robot operator permissions and rules, stored and archived the navigated results and printed reports and stored in an independent database.
\end{abstract}

Copyright $@ 2019$ Institute of Advanced Engineering and Science. All rights reserved.

\section{Corresponding Author:}

Nabeel Salih Ali,

Information Technology Research and Development Centre,

University of Kufa,Kufa, P.O. Box (21), Najaf Governorate, Iraq.

Email: Nabeel@uokufa.edu.iq

\section{INTRODUCTION}

Different technological terms such as Telecommunications, Internet of Things (IoT), and robotics have been considered a vital part of our daily activities [1]-[3]. Although its advances and limitations, innovative tech can be solved fundemental issues and save lives for many people for political or financial purposes [4]-[7]. In the electronic era, speed, flexibility, and automation are major defiance that is enabling researchers to meet the challenges of the society against the quick development of the techs [8-11]. Robotics have been becoming dynamically significant for several standard applications [12]-[14]. Applications such as military, Salvage and Urban Hunt. Due to its human reduction activities in a severe environment [15]-[17]. Effectiveness metal and landmine detections are two vital research areas that still considered more attractive to researchers due to investing tech [18]-[20]. According to Landmine and Cluster Munition Monitor report in 2014, more than 110 million landmines in the ground scattered in 68 countries [21]. So, landmines and metal detector robots are the advances innovation of the crucial and most dangerous part of the humanitarian detection process [22]. Multi-sensor robot, path planning algorithm, and vehicle-mounted sensors are different strategies that used to search and detect mines [23]-[25]. These applications are safer and more efficient due to they provide a safe route for the soldiers through minefields [26]. Robotics, communication, and data analysis have been advanced with high-speed achieved in landmine detection domain [1]. Data management, analysis, and archiving for the detection, navigating and mapping area will open the door to 
more deployed possibilities for furthermore mapping and detection areas. Automation mechanism presents easier and faster scanning process due to it provides the guarantee to more rapid the scanning processing and investigation automatically. As well, it fits more deployed capabilities based on the big data received to disclose and recognize metals and mines from other objects.

Several attempts by authors have been made for research and development perspectives concern assist or automate human deminers in the humanitarian demining and scanning process to reduce efforts, time, cost, human risks and dangerous issues by proposing diverse efficient and suitable robotics landmine detection with several sensors design [27]-[28]. Recent robotics research involves various trends such as sensor technology, Ground-Penetrating Radar (GPR), Electromagnetic Induction (EMI), Nuclear Quadrupole Resonance (NQR) and so on to perform multi-functions such as detection, deactivating, extracting, and demining for explosive devices, cluster munitions, landmines as well avoiding obstacles; These trends can deploy in diverse soil kinds such as foliage, dry and desert soil based on Aerial and wheel techniques for landmine detection [29]-[31]. Proposed Marwa which is a landmine detecting robot. Marwa is supported with a robotic arm whose payload a metal detector and provided visual information to be a visual servoing system to benefit researchers with limited resources to work in this area [32]. Also, presented a metal mine signals for detection purpose to estimate the depth of the metallic targets. The metal mine detector using robotic manipulator to take advantage of high precision scanning of the minefield; the proposed detector was fast and accurate in Humanitarian Demining; As well, it pre-build library containing data of many targets at different postures and depths [33]. Whereas, a remote robot is conducted to identify personnel landmines in diverse domains. The detector was obtained (87.5\%) accuracy from a set of 8 different materials in identifying mines. Also, the detection system can be implemented in different terrains [34]. introduced three-way to avert the issues of using one technology by using data fusion of multi-sensor system based on developing decision level fusion to decrease false alarms [26]. While, presented multi-sensor data-fusion approach by a highaccuracy geo-referencing the field-data acquired by multiple platforms to localize and detect purposes in landmines domain [24]. Likewise, introduced a novel landmine detection sensor that based on the principle of 2-DOF vibration absorber system.

The proposed new sensor gives the sensitivity of $1559 \mathrm{~Hz} /(\mathrm{MN} / \mathrm{m})$ and linearity better than (95\%) [35]. Besides, in 2016, Hank and Haddad presented a mobile robot for autonomous-navigation problem based on a hybrid approach; the produced navigator subjected to perform an emergency task with shorter execution times [36]. As alongside, designed and fabricated an efficient wireless controlled robot to detect landmine in defence fields as well avoiding the obstacles robustly; H-Bridge module used to control the robot wheels and wireless camera added to capture and located off the robot destination [37]. Also, proposed a low-altitude autonomous flight to detect landmines; the system called Backstepping+DAF which is an integrated system architecture based on lightweight GPR [38]. As well, a hybrid platform introduced by Ghareeb et al. (2017), speed data transferring and transmission quality to improve central unit destination that is based on web server and a database server applications to store data regarding the navigation field for current mapping and detection or future investigation purpose [1].

In this research, highlights on metal detection issues. Also, the capability to detect mines, avoid obstacles. The proposing system using the concept of metal detection sensor and ultrasonic sensor, wireless communication via controlled robotics vehicle remotely for flat surfaces and dry, desert soils to design and fabricate detect metals without human harm. Also, proposed an integrated management and achieving system to control, manage and stored the navigation results with online structured query language databased registry on a server side to give high-security system. As well, conduct a comparative analysis of the proposed robot detection systems and focus on their main findings and systems features like detection, controlling, management, and archiving information in the database server for future mapping and navigation. The rest of the article is structured as follows: In Section 2, describes hardware and software that required to produce the controlling, management, and archiving system that includes three subsections are metal detector robot architecture, management and controlling the metal detector robot, and archiving database system. Section 3 presents all steps and processes to implement the proposed detection system. Besides, results and discussion will be included in Section 4. Whereas, Section 5 gives a comparison of the works that have been done to detect landmine detection. Finally, Section 6 concerns with conclusions and future directions.

\section{RESEARCH METHODOLOGY(MATERIALS AND METHODS)}

In this section, describes the methodology used in this research, explaining the particular materials, methods, and techniques used that depend on the sensor-based technology. The research methodology presents the design and development of the proposed system. The methodology which used in this research comprises of three phases: metal detector, control and management, integrated database system phase, as shown details in Figure 1. 


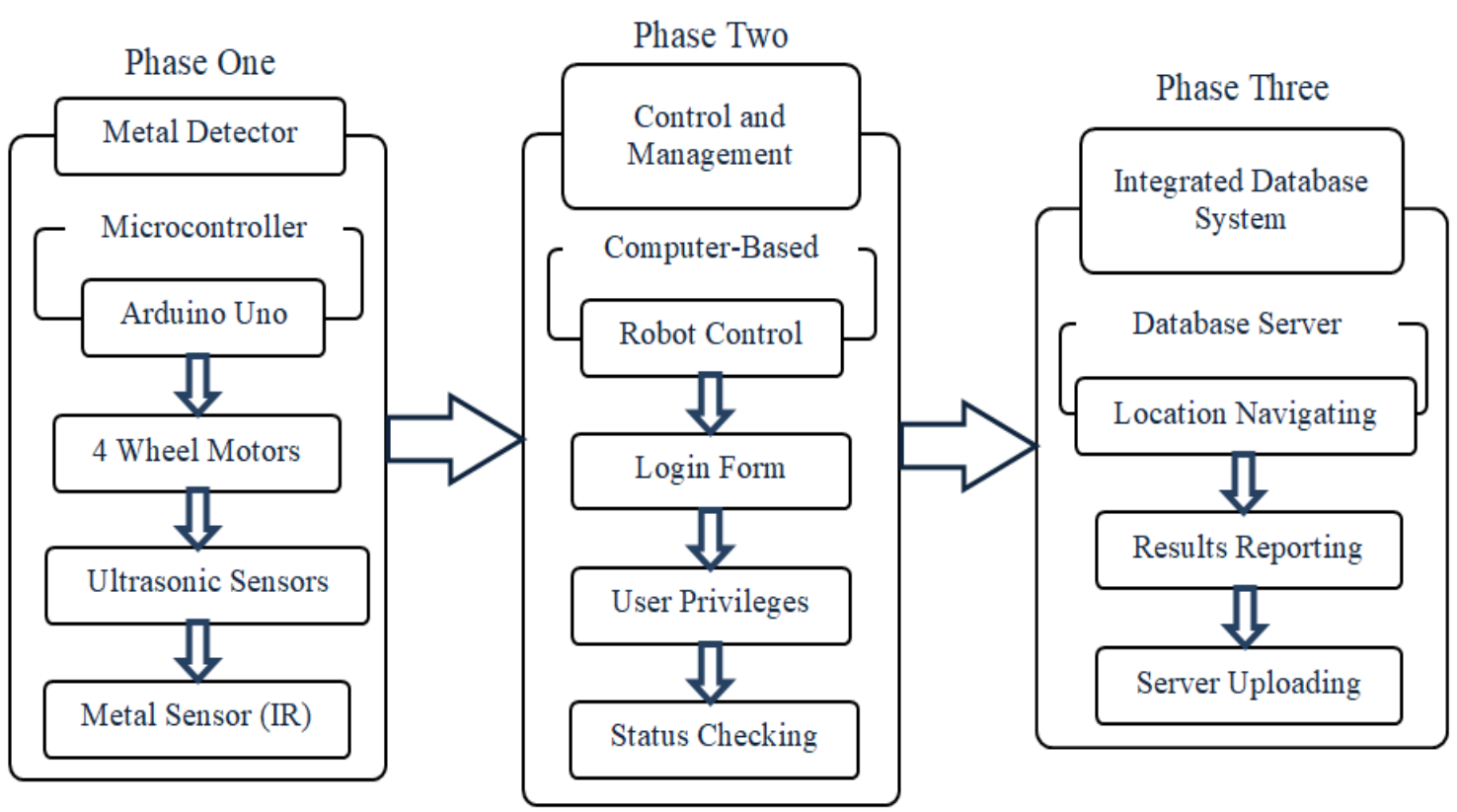

Figure 1. Metal detector robot methodologies phases

\subsection{Metal detector robot architecture}

The detector aims to disclose an underground metal surface for a particular navigation area. This section describes materials and methods of the metal detector robot architecture. The proposed robot detector involves hardware and software components that needed to implement the robot navigator. These elements have been chosen based on cost-effectiveness, usability, availability in the fabricated region, and ease of programming metrics. The hardware components have required for implementing and testing the computer application based on controlling the detector. While Arduino Uno microcontroller boards as the brain of the system detection. Different sensors deployed for detecting mines or metals, avoiding obstacles in real-time settings such as metal detection sensor and ultrasonic sensors. Figure 2 shows the block diagram of the proposing metal detection system.

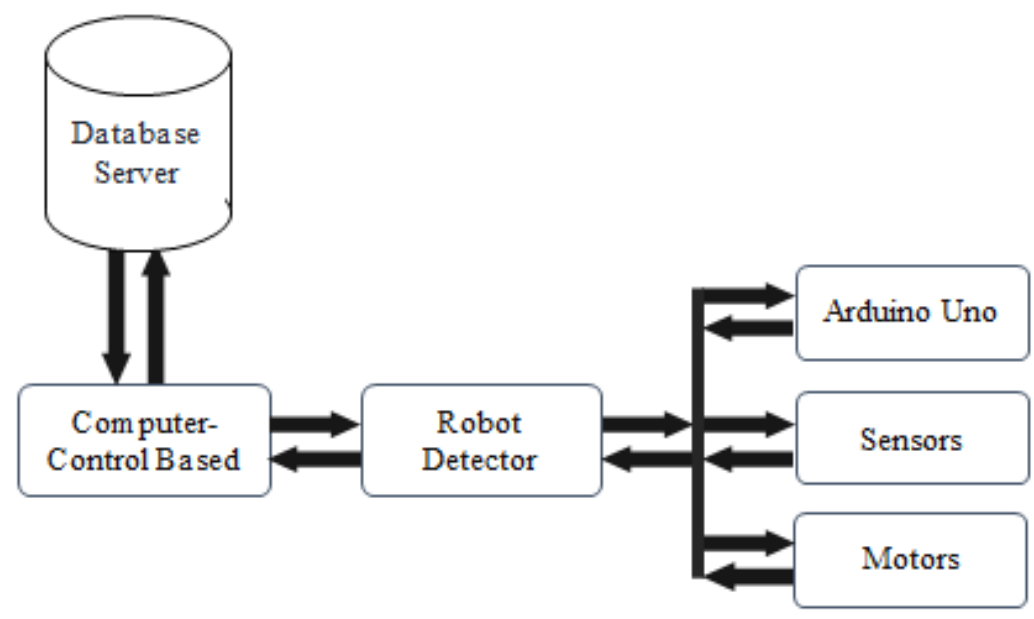

Figure 2. Block diagram of the proposed metal detection, archiving, and management system

A microcontroller Arduino UNO board (ATmega328P) used as a brain to give instructions and analysis the results. Arduino microcontroller involves physical programmable circuit board and a piece of software that it runs on computer to be used for writing and uploading computer code to the physical board). Uno processes the received data from the computer, run the sensors, receive their results and analysis to send 
them to the computer, run the motors and move the robot to the targeted place and stop the robot in case of a metal or obstacles disclosed and send an alert to the computer. Besides, the middleware between the computer and robotic circuit is a Bluetooth technology which is adapted to be the transmission channel between system peripherals wirelessly. Bluetooth is a short-range wireless connection between computers and other devices. Figure 3 presents a flowchart that clarifies sending and receiving information mechanism.

On the other hands, the robot used ultrasonic sensor (HC-SR04) which radiated ultrasound to detect and avoid obstacles. Figure 4 illustrates the robot scenarios of metals and obstacles revealed. The ultrasonic sensor (HC-SR04) is used to find out the distance between the robot and obstacles that are positioning in front of its route that controlled via Arduino. Obstacles distance have calculated based on the difference between time sending and receiving the radiated ultrasound by the ultrasonic sensors when a particular obstacle is discovered. The mechanism of disclosing obstacles are used to decide the best route when there are many routes in the navigation and mapping area.

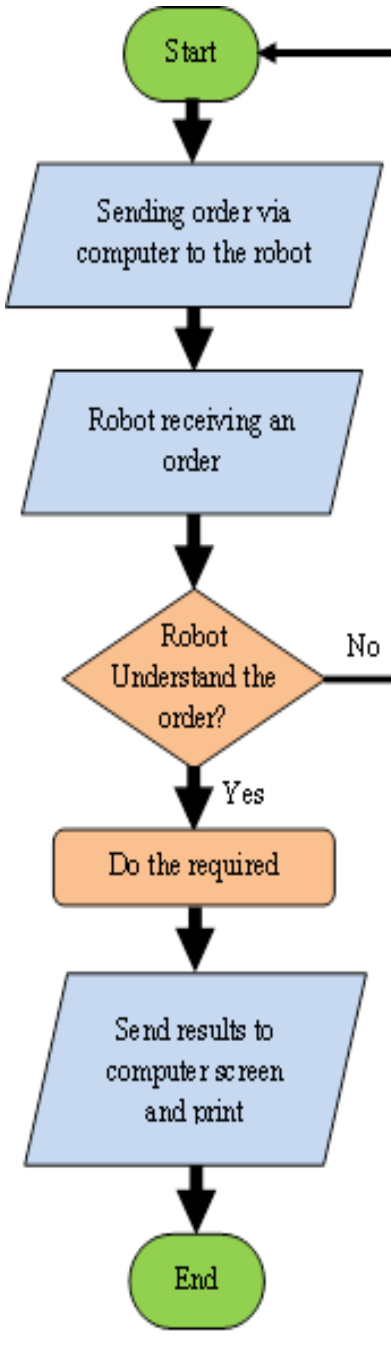

Figure 3. Flowchart of sending and receiving information mechanism

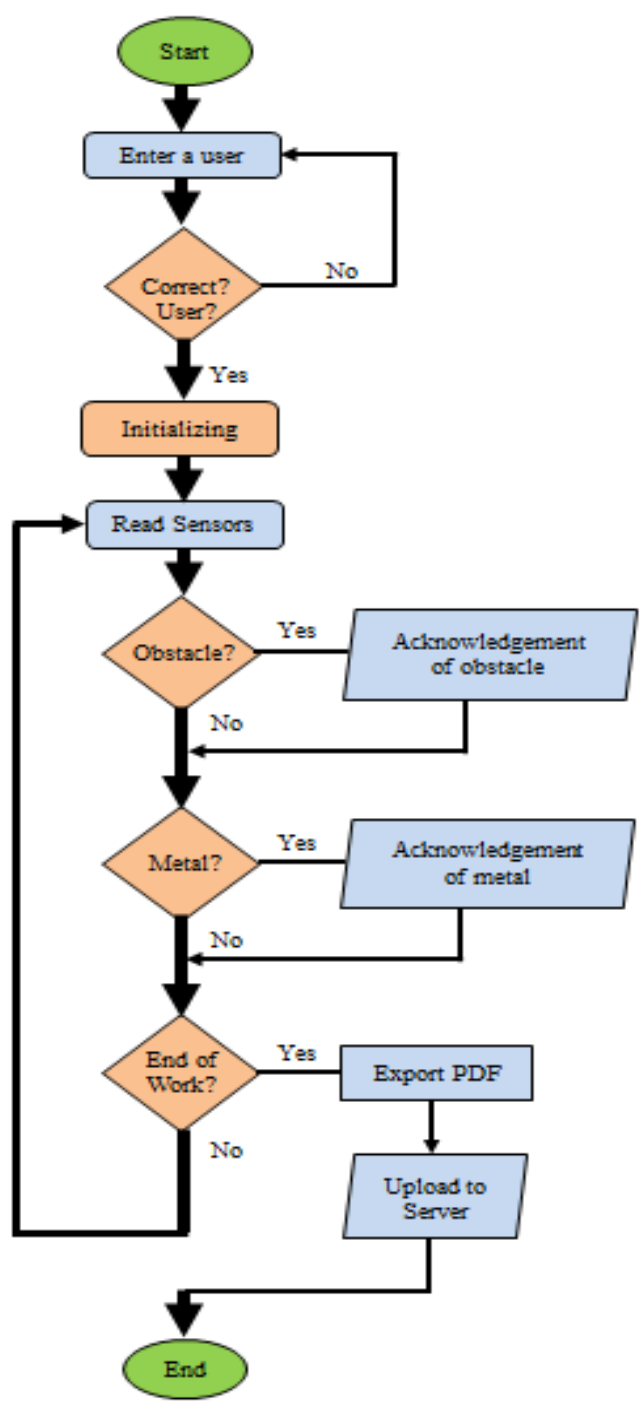

Figure 4. General flowchart of the metals and obstacles detector robot

\subsection{Management and controlling the multi-sensor robot}

Computer application based is designed via using a Visual C\# program to control the robot and move it to the current place for detecting the metals and obstacles consecutively. And archive all the results in a database as reports as well uploaded to a server for future navigated purposes. Where the robot received and transmitted information via the computer application by Bluetooth wireless technology. This mechanism 
is achieving the interface between Arduino Uno (open source computer hardware), C\# (multi-paradigm programming language) and SQL server (Structured Query Language). As we shown in Figures 3 and 4. Hence, detector processes the received data from the computer, run the sensors, receive their results and analysis to send data to the computer, run the motors to move the robot to the targeted place and stop the robot in the case of a metal or the fact of obstacles any? And send an alert to the computer. Admin and users can be accessed and managed the detection system via login form with different permission. The permission of administrator is having full accessing to the database, adding, deleting and editing the user (worker) or even the reports, while the user (worker) can only start the robot and upload reports to the database. A computerized database has been linked to an electronic filing cabinet of data arranged for easy access or a specific purpose. Figure 5 presents the login form with its information required.

After entering to the robot control panel via the login form that appeared to check the details of the user and the user will enter the location that is searching it to archive them to the database. The first part of the robot control panel is the connection part of the detector by writing the COM number of Bluetooth. If the robot is switched off or there is any problem, an alert will be presented as shown in Figure 6.

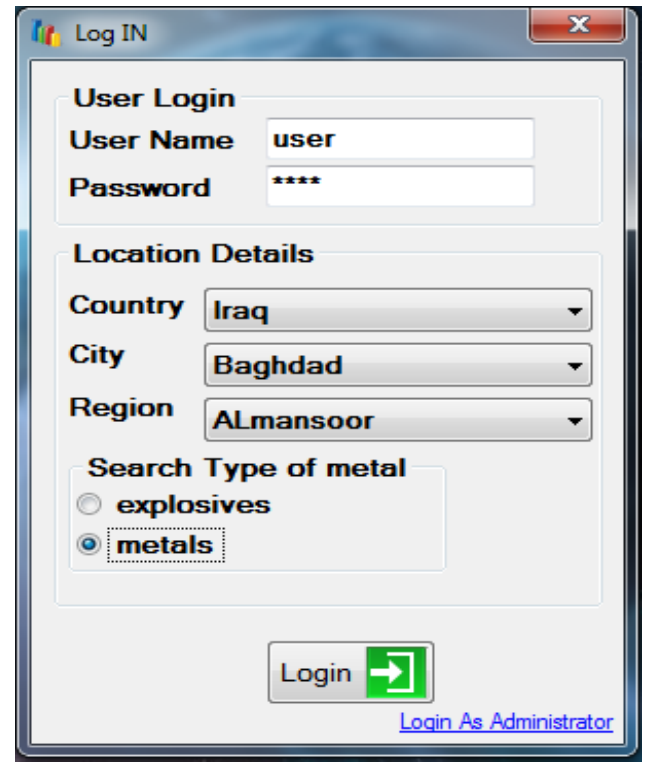

Figure 5. Login form of the controlled system

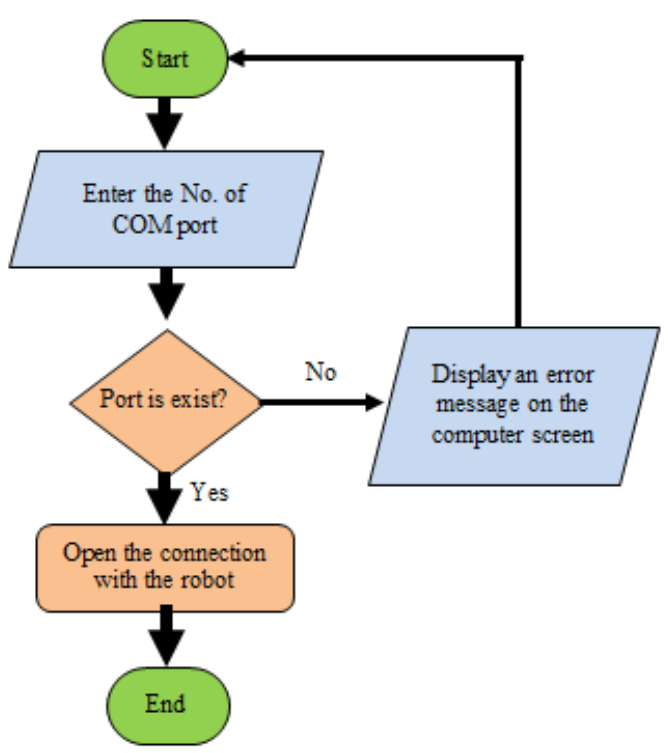

Figure 6. Flowchart of the connection mechanism

The status function on the robot will send some details to the computer, all these details will analyse by the system and shown on the robot control panel, these details like battery level and status screen will display information if the robot detects a metal or detect an obstacle and other things to be easy to understand the status of the robot at this time. There are some buttons is used to give an order to the robot for moving perspectives, and searching on the metal, and change the speed of the robot. If the robot detects obstacles, the testing way function is used to see the best way if there is more than one way to select. Figure 7 presents the connection panel and their controlled data and buttons.

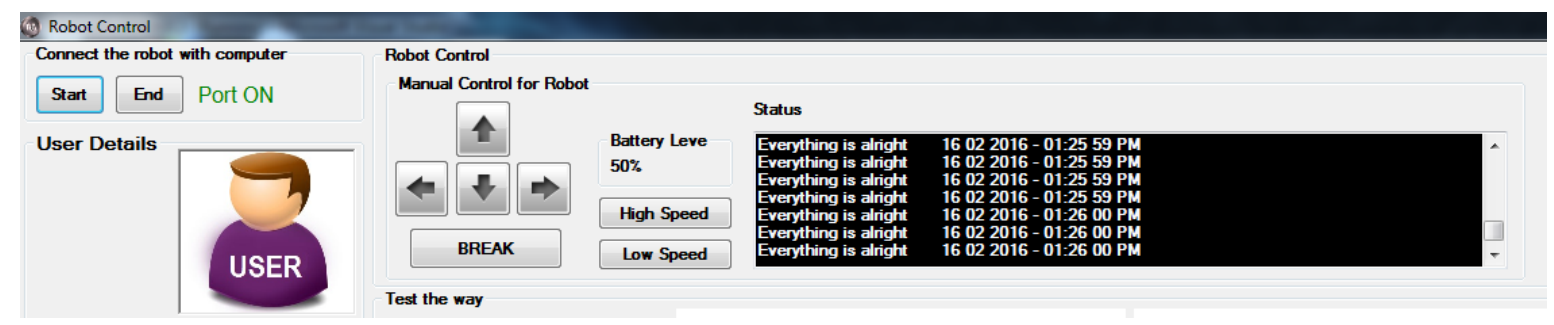

Figure 7. Connection and controlled panel 
There is another control panel that just the admin of the system can enter it, as shown in Figure 8. In this panel the admin can add and remove the users, can add and remove the location of searching and can explain all the reports and search for the desert report that saved in the database before. Hence, the user cannot write any searching region but the admin can add them and the user just selected.

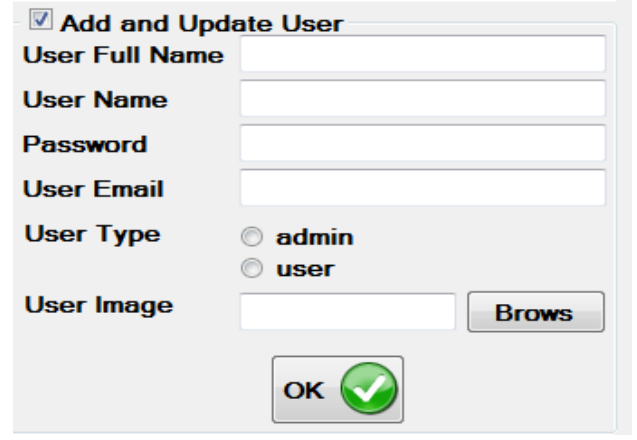

(a)

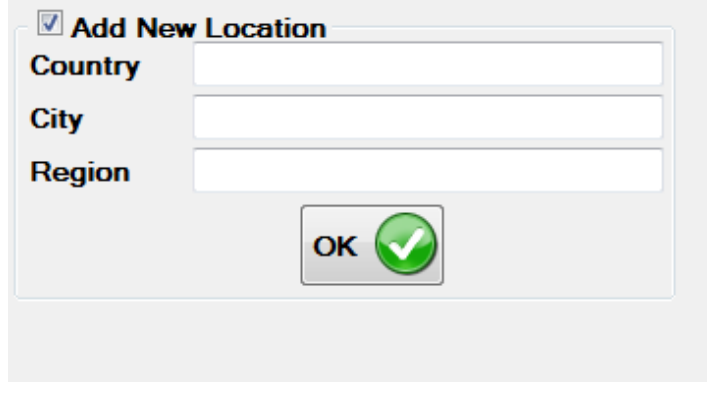

(b)

Figure 8. Administrator privileges and functions

\subsection{Archiving database system of the metal detector robot}

The system contains a database scheme to store and archive the detector results, there are two types of users for the system, manager or admin and robot worker or operator, the manager can use the robot and show the results that saved in the database, but robot worker can only use the robot and upload reports files to the database server. An integrated database system is used to set of permissions and rules for employees by managers as well as archiving the reports and the results in an independent database registry. Figure 9 presents an example of the printed report as a PDF file.

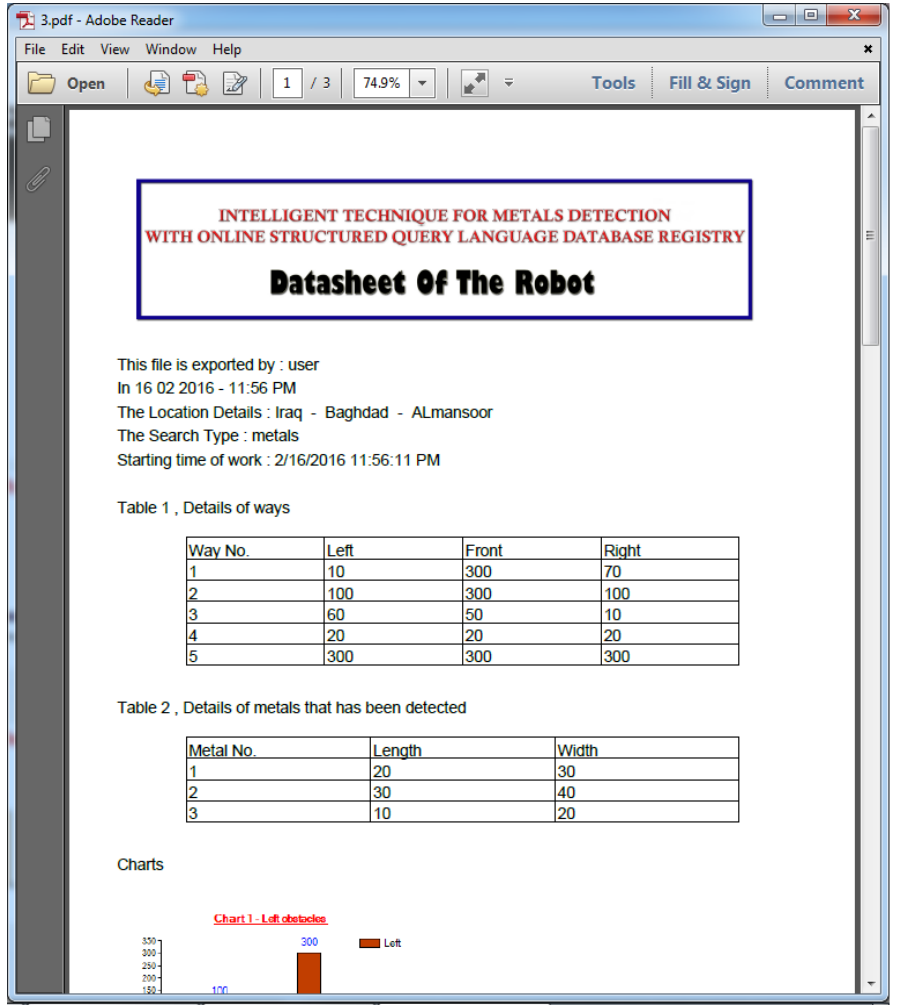

Figure 9. Printing the navigation results reports 
After the robot worker finishes his work, then be able to export and saved charts, tables and all results as PDF file like a report. And upload the PDF report and results to the database server to store, the user can only upload the data to the server but the admin can access to the database and display results, as well he can perform to add and edit users and a particular location. If the admin removes all users, there is a default user the admin cannot remove him to save the system from full down. Figure 10 displays information regarding navigation location and their existing reports archived. This information can be edited, updated, deleted via the admin of the system only.

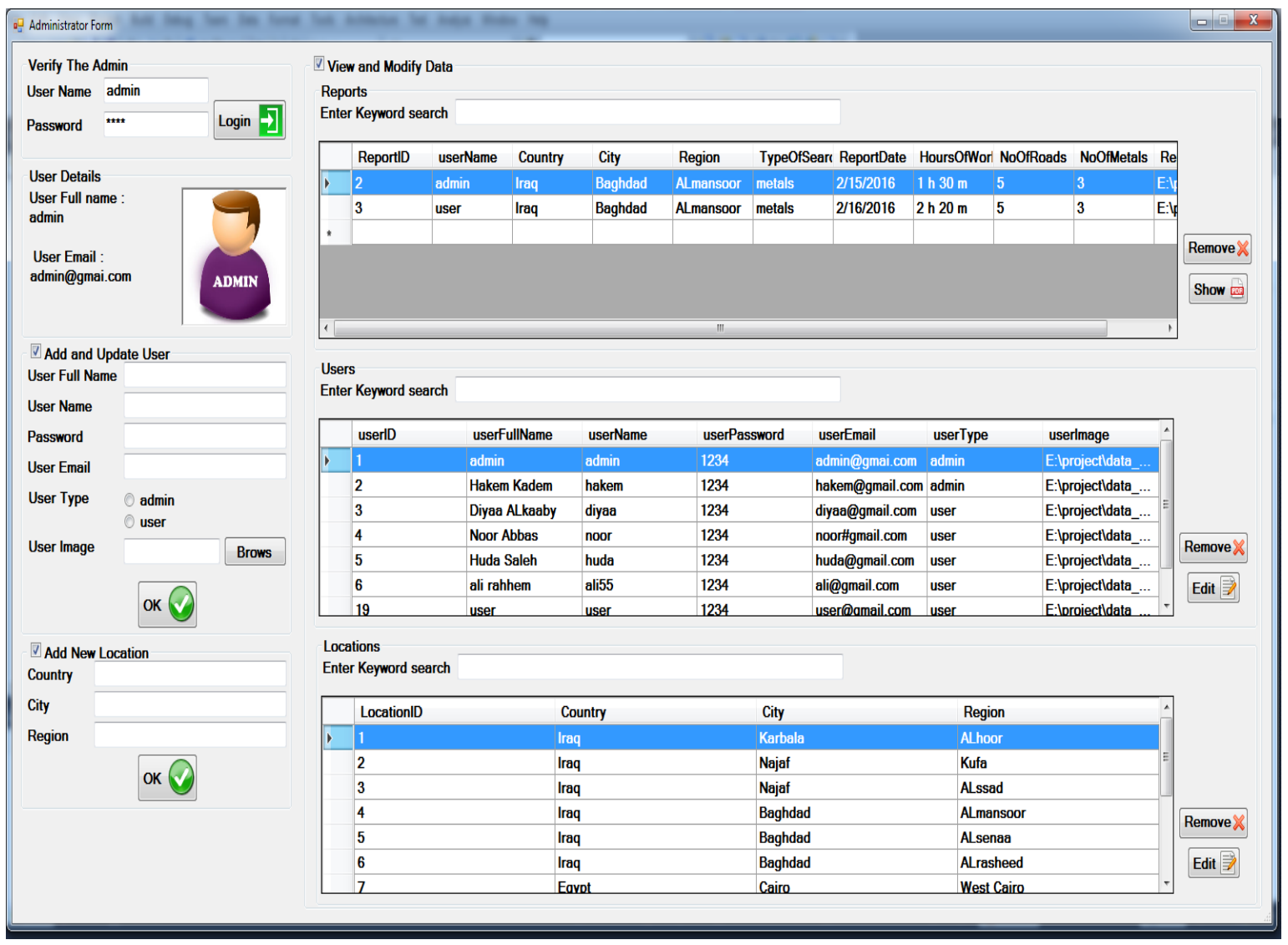

Figure 10. Admin control robot panel

\section{IMPLEMENTATION OF THE PROPOSED CONTROLLING, MANAGEMENT, AND ARCHIVING SYSTEM}

Design a robot with complete controlling, management and archiving system consists of the admin and users. They can use the robot for detecting minerals that subsurface. Therefore, the information that comes from the robot to the computer, it collects data from the report, and upload to the database to determine the best report by the manager in charge of the disclosure. The robot gets some orders from the computer that connected with it wirelessly, the exchange of information between the robot and the computer is done via Bluetooth technology by an algorithm. Figure 10 displayed the robot control panel when a particular admin logged. The proposed system can be used in the detection operations of metals, and save all operations information carried out by the robot to the database server device that gives high security for the system. So the most important advantages of this system are supporting the prospectors to locate some details of metals, design an integrated system to archive reports and results. Figure 11 shows the metal detection results for five ways. 


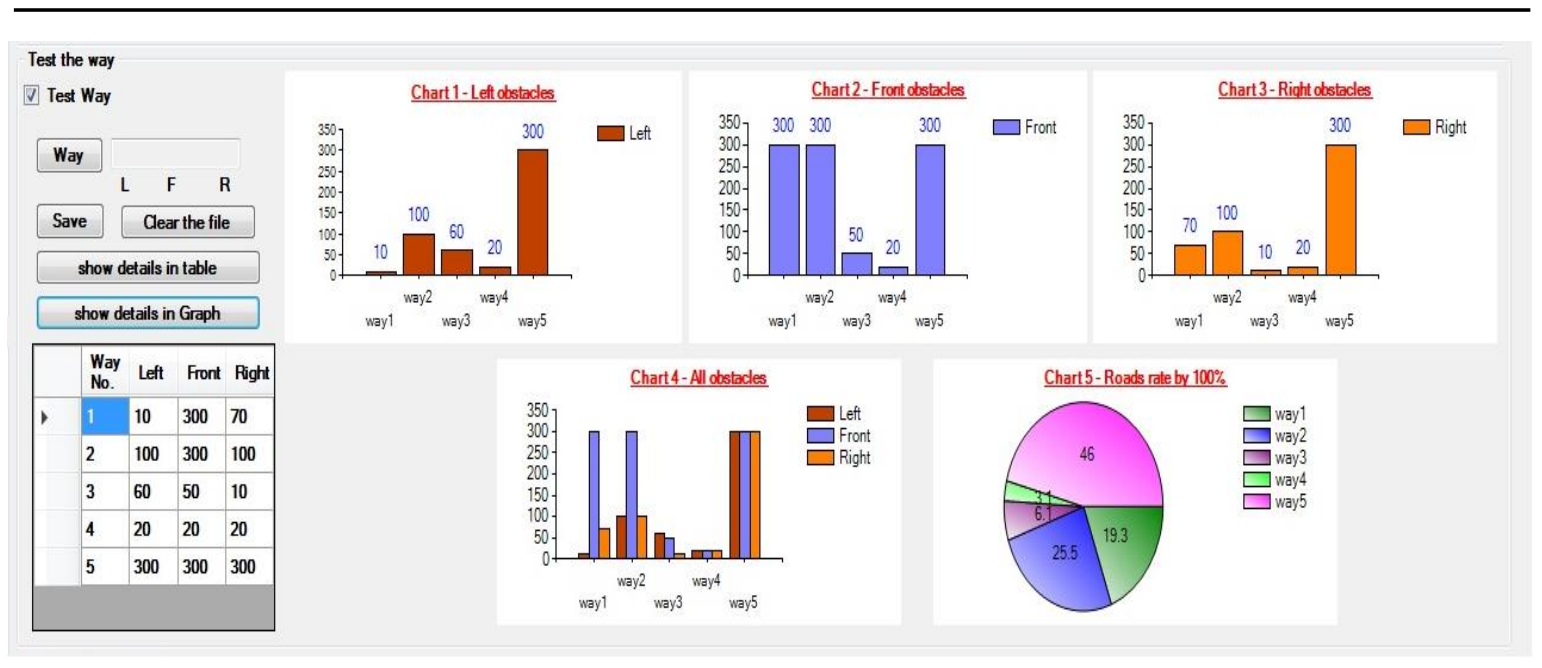

Figure 11. Metal detection system testing results

\section{RESULTS AND DISCUSSIONS}

Three main points that are concentrated are, 1) Design a robot that carries the sensor of the metals and obstacles, 2) Connect the robot wirelessly with the computer-based application for controlling and management purposes via creating a full system to control the robot, and 3) Manage all the results in reports and save them in the database server. As we show in Figure 12 which describes an overview of the proposed multi-sensor robot system.

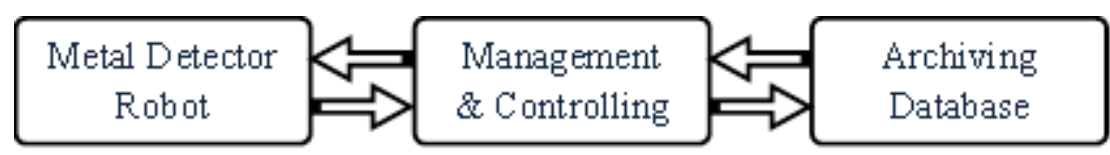

Figure 12. Overview diagram of the proposed detection system

Based on Figure 11, the system analyzed the results and displayed in multi-chart. Whereas, chart 1 lists obstacles distances at the left positioning of the front of the robot detector path. So, there is an obstacle which it distance $(10 \mathrm{~cm})$ from the robot in the way No. 1 and there is an obstacle at a distance $(100 \mathrm{~cm})$ from the robot in the way No. 2 and the rest obstacles distances in reminder five ways as follows respectively (60 $\mathrm{cm}, 20 \mathrm{~cm}, 300 \mathrm{~cm}$ ). As a long side, chart 2 presents the distances of the detected obstacles via Ultrasonic sensor in the front of the detector positioning mapping area. Whereas, the chart 2 contains obstacles distances of the five ways values as follows consecutively $(300 \mathrm{~cm}, 300 \mathrm{~cm}, 50 \mathrm{~cm}, 20 \mathrm{~cm}$, and $300 \mathrm{~cm})$. Likewise, chart 3 views the distances of the obstacles at the right of the front of the robot navigator. The five ways values were $(70,100,10,20,300) \mathrm{cm}$.

Depend on the results in chart 2, there is an obstacle on distance $(300 \mathrm{~cm})$ from the robot in the way No. 1 and there is an obstacle at a distance $(300 \mathrm{~cm})$ from the robot in the way No. 2 and there is an obstacle at a distance $(300 \mathrm{~cm})$ from the robot in the way No. 5 . Whilst, in chart 1 only way No. 5 has an obstacle distance is $(300 \mathrm{~cm})$ also in chart 3 , way No. 5 has an obstacle distance $(300 \mathrm{~cm})$.

The experiment results of the proposed metal detector robot present the detector is capable of detecting more than one metals kinds such as iron, silver, and copper efficiently in the navigation area. Based on Figure 11, there are five ways discovered by the detector in the mapping field. The results found by the ultrasonic sensors that connected with detector robot to disclose the obstacles. The results that are obtained from sensing paths are shown in charts to present the distance of the discovered obstacles that positioning at the front of the robot and to make a better decision for which route is better and fast to navigate it shortly.

\section{COMPARISON OF THE PREVIOUS METAL DETECTION SYSTEMS AND THEIR MERITS}

Diverse researchers have been attempted on proposing and developing detection, deactivating, extracting, and demining for explosive purposes. These efforts produced new and novel solutions in the 
humanitarian demining and scanning process to reduce efforts, time, cost, human risks and dangerous issues [29], [31]. One of the solutions was by proposing diverse efficient and suitable robotics landmine detection with several sensors design [27], [28]. GPR, EMI, NQR, and sensor technology are the common trends in the robotics research recently. Table 1 presents a comparison of the previous detection systems in landmine and metal applications. It consists of several terminologies such as system features and functionalities such as detection, controlling and management end, and the database storing and archiving as well as highlights on their main findings.

Most of the proposed detector systems have controlled and managed their detection robot except [35], on the other hands, three only names [24], [32], and [1] have conducted a database end to store information of the navigated areas for future mapping. As alongside, two systems only that namely [26], and [36] are used path planning algorithms and optimal motion planning techniques in their detectors. Whereas, most of the proposed systems are based on vehicle-mounted sensors (remote sensing) except two only that are [35] and [38]. Fusion methods which include multi-level such as decision level, feature level, and data level are used a few system detectors like [24], [26], and [36].

Table 1. Comparison of the Previous Detection Systems

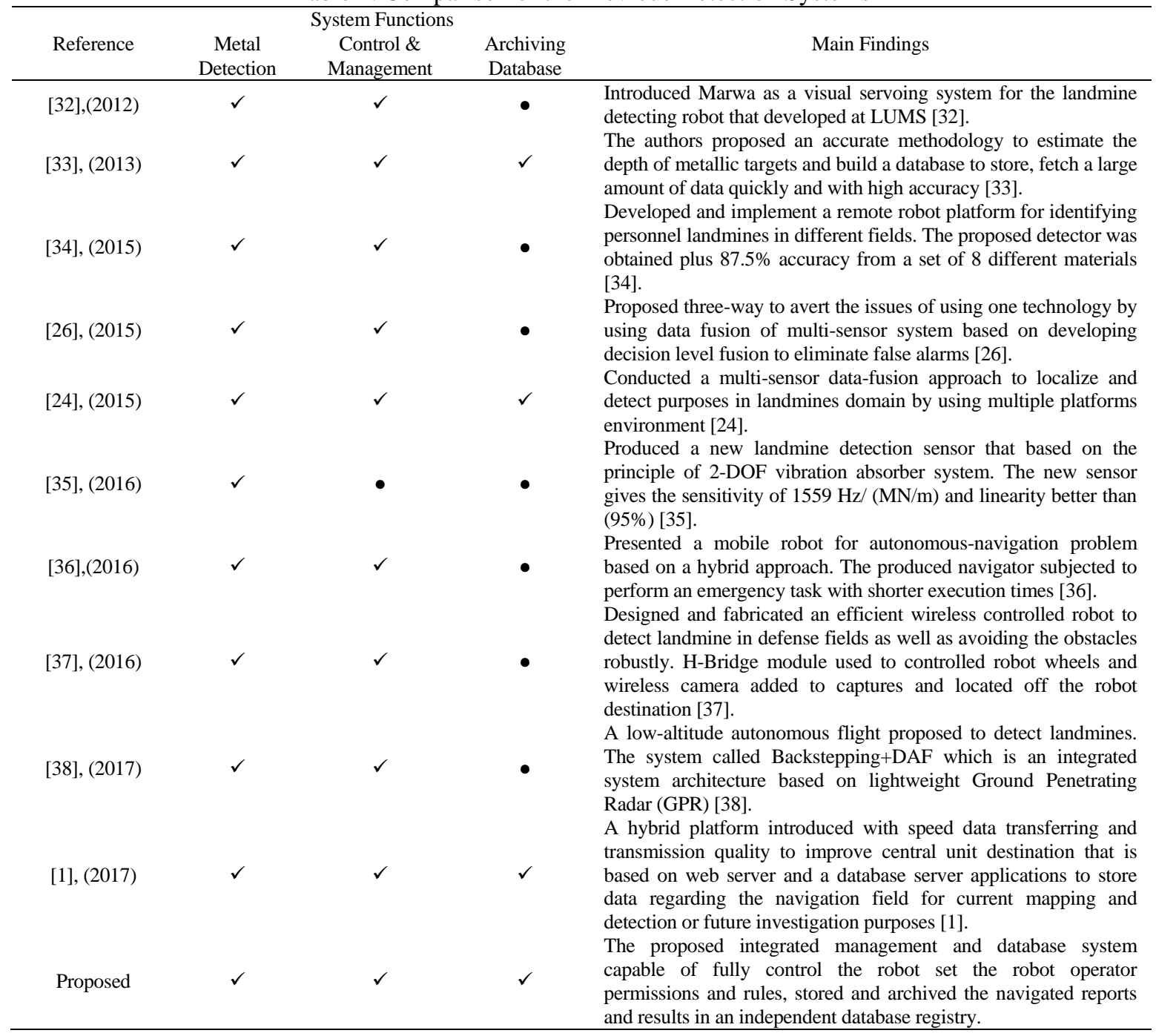

\section{CONCLUSIONS AND FUTURE DIRECTIONS}

The main idea is to design and implement a prototype of an efficient low-cost automated mine detector that will replace the current employed human detectors in the mission of detecting and extracting mines in a suspected area of land. As well, communication and data analysis in the domain of landmine detection. The detector wirelessly communicates with a server to transmit and store the detected information such as the location of the metal object and captured images of the land where does it exist. Designing an

Management and achieving system for metal detection robot using wireless-based... (Hakim Adil Kadhim) 
integrated system which consists of a simple robot provides with a metal detector and uses Bluetooth technology to communicate with its software inside the computer. The system comprises two types of users. An admin can control the system, and a user can only control the robot. In the intelligent algorithm, the robot can discover the obstacles in the front of it, so if there is more than one way to enter the searching place, the robot can detect the best way that has the lowest obstacles. The robot will send the data to the computer, so the system displayed the received data from the robot and analyze them. All results will be exported as PDF file and upload to an online database that connected with the system to archive the results. In the future perspectives, intend to develop the proposed system to perform different perdition objects in the mapping and navigated areas based on supported data set. As well, test the system with robot mobile-based.

\section{REFERENCES}

[1] Ghareeb, M., Bazzi, A., Raad, M., \& AbdulNabi, S, "Wireless robo-Pi landmine detection. In Landmine: Detection, Clearance and Legislations (LDCL)," 2017 First International Conference on (pp. 1-5). IEEE, April 2017.

[2] Craig, J. J., "Introduction to robotics: mechanics and control," Upper Saddle River, NJ, USA: Pearson/Prentice Hall, Vol. 3, pp. 48-70, 2005.

[3] Olley, G. S., and Pakes, A., "The dynamics of productivity in the telecommunications equipment industry" (No. w3977). National Bureau of Economic Research, 1992.

[4] Li, Shelei, Xueyong Ding, and Tingting Yang. "Analysis of Five Typical Localization Algorithms for Wireless Sensor Networks." Wireless Sensor Network 7.04: 27, 2015.

[5] Magrabi F, Aarts J, Nohr C, et al., "A comparative review of patient safety initiatives for national Health information technology," Int J Med Inform; 82:e139-48, 2013.

[6] Pugh, J., and Martinoli, A., "Inspiring and modeling multi-robot search with particle swarm optimization," In Swarm Intelligence Symposium, 2007. SIS 2007. IEEE (pp. 332-339). IEEE, April 2007.

[7] Rjeib, H. D., Ali, N. S., Al Farawn, A., Al-Sadawi, B., and Alsharqi, H., "Attendance and Information System using RFID and Web-Based Application for Academic Sector," International Journal of Advanced Computer Science and Applications (IJACSA), 9(1). 2018.

[8] Suresh, K., Vidyasagar, K., and Basha, A. F., "Multi Directional Conductive Metal Detection Robot Control. International Journal of Computer Applications, 109(4), 2015.

[9] Ambruš, D., Vasić, D., and Bilas, V., "Robust estimation of metal target shape using time-domain electromagnetic induction data," IEEE Transactions on Instrumentation and Measurement, 65(4), 795-807, 2016.

[10] Albert, F. Y. C., Mason, C. H. S., Kiing, C. K. J., Ee, K. S., and Chan, K. W., "Remotely operated solar-powered mobile metal detector robot," Procedia computer science, 42, 232-239, 2014.

[11] Ali, N. S., Alyasseri, Z. A. A., and Abdulmohson, A., "Real-Time Heart Pulse Monitoring Technique Using Wireless Sensor Network and Mobile Application," International Journal of Electrical and Computer Engineering (IJECE), 8(6), 2018.

[12] Alauddin, T., Islam, M. T., and Zaman, H. U., "Efficient design of a metal detector equipped remote-controlled robotic vehicle," In Microelectronics, Computing and Communications (MicroCom), 2016 International Conference on (pp. 1-5). IEEE, January 2016.

[13] Makki, I., Younes, R., Francis, C., Bianchi, T., and Zucchetti, M., "A survey of landmine detection using hyperspectral imaging," ISPRS Journal of Photogrammetry and Remote Sensing, 124, 40-53, 2017.

[14] Sudac, D., Majetic, S., Nad, K., Obhodas, J., and Valkovic, V., "Improved system for inspecting minefields and residual explosives," IEEE Transactions on Nuclear Science, 61(4), 2195-2203, 2014.

[15] Arvinth, R., "Autonomous Navigation Robot for Landmine Detection and Fire Extinguisher Application using Zigbee Protocol,"

[16] Portugal, D., Cabrita, G., Gouveia, B. D., Santos, D. C., and Prado, J. A., "An autonomous all terrain robotic system for field demining missions," Robotics and Autonomous Systems, 70(C), 126-144, 2015.

[17] Srivastava, A., Vijay, S., Negi, A., Shrivastava, P., and Singh, A., "DTMF based intelligent farming robotic vehicle: An ease to farmers," In Embedded Systems (ICES), 2014 International Conference on, pp. 206-210. IEEE, July.2014.

[18] Zin, Z. A. M., Ali, F., and Ab Kadir, D., "Automatic and Obstacle Avoidance in Metal Detector Robot," Journal of Computing Technologies and Creative Content (JTeC), 1(1), 27-29, 2016.

[19] Boukadida, S., Gdaim, S., \& Mtiba, A., "Sensor Fault Detection and Isolation Based on Artificial Neural Networks and Fuzzy Logic Applicated on Induction Motor for Electrical Vehicle," International Journal of Power Electronics and Drive Systems (IJPEDS), 8(2), 601-611, 2017.

[20] Cluster Munition Monitor, Aug 2014, [online] Available: http://archives.the monitor.org/index.php/LM/OurResearchProduct/CMM14.

[21] Salam Kadhim, Dheyaa Alhelal, Nabeel Salih Ali, "Energy Efficient Cluster Based Routing Protocol for Dynamic and Static Nodes in Wireless Sensor Network," TELKOMNIKA (Telecommunication Computing Electronics and Control), 16(5). 2018.

[22] Ambrus, D., Vasic, D., and Bilas, V., "Model-based target classification using spatial and temporal features of metal detector response,” In Sensors Applications Symposium (SAS), 2015 IEEE, pp. 1-6. IEEE, April, 2015. 
[23] S. Larionova, A. de Almeida, and L. Marques, "Using robots in hazardous environments: Landmine detection, demining and other applications," Woodhead Publishing, ch. Sensor Fusion for Automated Landmine Detection with a Mobile Robot, pp. 147-188, 2011.

[24] Prado, J., and Marques, L., "Multi-sensor and multi-platform data fusion for buried objects detection and localization," In Autonomous Robot Systems and Competitions (ICARSC), 2015 IEEE International Conference on (pp. 186-191). IEEE, April, 2015.

[25] Bouguenna, I. F., Azaiz, A., Tahour, A., and Larbaoui, A., "Hybrid Fuzzy Sliding Mode Speed Control for an Electric Vehicle Drive," International Journal of Power Electronics and Drive Systems (IJPEDS), 8(3), pp. 1050-1061, 2017.

[26] Ismail, A., Elmogy, M., and ElBakry, H., "Landmines detection using low-cost multisensory mobile robot," Journal of Convergence Information Technology, 10(6), 51, 2015.

[27] Habib, M. K., "Humanitarian demining: reality and the challenge of technology-the state of the arts," International Journal of Advanced Robotic Systems, 4(2), 19, 2007.

[28] Estremera, J., Cobano, J. A., \& De Santos, P. G., "Continuous free-crab gaits for hexapod robots on a natural terrain with forbidden zones: An application to humanitarian demining," Robotics and Autonomous Systems, 58(5), 700-711, 2010.

[29] Nuzzo, L., Alli, G., Guidi, R., Cortesi, N., Sarri, A., and Manacorda, G., “A new densely-sampled ground penetrating radar array for landmine detection," In Ground Penetrating Radar (GPR), 2014 15th International Conference on (pp. 969-974). IEEE, June, 2014.

[30] Elnabi, T. A. H., "Design And Implementation of a Mine Sweeping Machine," Doctoral dissertation, University of Khartoum, 2017.

[31] Florez, J., and Parra, C., "Review of sensors used in robotics for humanitarian demining application". In Robotics and Automation (CCRA), IEEE Colombian Conference on (pp. 1-6). IEEE, September, 2016.

[32] Manzoor, T., Munawar, A., and Muhammad, A., "Visual servoing of a sensor arm for mine detection robot marwa. In Robotics," Proceedings of ROBOTIK 2012; 7th German Conference on (pp. 1-6). VDE, May, 2012.

[33] Kaneko, A. M., Endo, G., and Fukushima, E. F., "Landmine buried depth estimation by curve characterization of metal mine detector signals," In Intelligent Robots and Systems (IROS), 2013 IEEE/RSJ International Conference on (pp. 5327-5332). IEEE, November, 2013.

[34] Casas-Diaz, C. A., and Roa-Guerrero, E. E., "Development of mobile robotics platform for identification of land mines antipersonal in different areas of Colombia," In Communications and Computing (COLCOM), 2015 IEEE Colombian Conference on (pp. 1-6). IEEE, May, 2015.

[35] Ali, H. F., El-Bab, A. M. F., Zyada, Z., and Megahed, S. M., "Parameter optimization of a novel contact sensor based on frequency response of 2-DOF vibration absorber system for landmine detection," In Robotics and Intelligent Sensors (IRIS), 2016 IEEE International Symposium on (pp. 96-102). IEEE, December, 2016.

[36] Hank, M., and Haddad, M., "A hybrid approach for autonomous navigation of mobile robots in partially-known environments," Robotics and Autonomous Systems, 86, 113-127, 2016.

[37] Farooq, W., Butt, N., Shukat, S., Baig, N. A., and Ahmed, S. M., "Wirelessly Controlled Mines Detection Robot," In Intelligent Systems Engineering (ICISE), 2016 International Conference on (pp. 55-62). IEEE, January, 2016.

[38] Colorado, J., Devia, C., Perez, M., Mondragon, I., Mendez, D., and Parra, C., "Low-altitude autonomous drone navigation for landmine detection purposes," In Unmanned Aircraft Systems (ICUAS), 2017 International Conference on (pp. 540-546). IEEE, June, 2017. 